\title{
Aspetti clinico-diagnostici e terapeutici nella gestione quotidiana dell'ipernatremia
}

\author{
Roberto Di Vito, Cosima Posari, Silvia Bucco, Martina Camplese, Federica Giunta, \\ Nicola Spetrino, Xhensila Grabocka, Maria Olimpia Longo, Simona Silvestri, \\ Giacinta Vigilante, Mario Bonomini
}

Scuola di Specializzazione in Nefrologia, Dipartimento di Medicina e Scienze dell'Invecchiamento, Università degli Studi G. D’Annunzio, Chieti-Pescara

\begin{abstract}
HyPERNATREMIA: CLINICAL, DIAGNOSTIC, AND THERAPEUTIC MANAGEMENT
Abstract. Disorders of water and sodium balance are highly common in hospitalized patients, especially in those critically ill. Hypernatremia, either due to sodium overload or hypotonic water depletion, strongly contributes to the morbility and mortality of this category of patients. Therefore, this disorder requires an early diagnosis and can be solved by an appropriate therapeutic intervention and treatment of the risk factors.
\end{abstract}

Key words: Hypernatremia, Water and sodium balance

Conflict of interest: None.

Financial support: None.

Accettato: 18 Giugno 2014

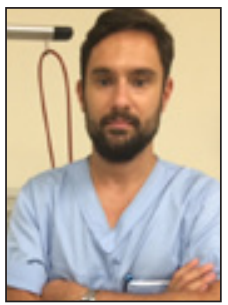

\section{Introduzione}

Lo ione sodio $\left(\mathrm{Na}^{+}\right)$rappresenta il principale catione del compartimento extracellulare ed è il principale determinante dell'osmolarità plasmatica, secondo la formula:

Roberto Di Vito

$$
\begin{gathered}
\text { osmolarità plasmatica }(\mathrm{mmol} / \mathrm{L})=1.863 \times \mathrm{Na}^{+}(\mathrm{mEq} / \mathrm{L}) \\
+ \text { glicemia }(\mathrm{mg} / \mathrm{dL}) / 18+\text { urea }(\mathrm{mg} / \mathrm{dL}) / 2.8
\end{gathered}
$$

In condizioni fisiologiche, l'osmolarità del liquido intracellulare e quella del comparto extracellulare sono mantenute costanti, mediante fini meccanismi di autoregolazione, intorno a valori di $280 \mathrm{mmol} / \mathrm{L}$. Eventuali variazioni di concentrazione dei soluti in uno dei due compartimenti vengono bilanciate da un movimento di acqua che avviene secondo il fenomeno di osmosi. I principali meccanismi di autoregolazione dell'osmolarità plasmatica sono:

- il sistema Renina-Angiotensina-Aldosterone, che determina ritenzione di acqua e sodio;

- i peptidi natriuretici, Atrial Natriuretic Peptide (ANP) e Brain Natriuretic Peptide (BNP), che inibiscono l'attività di renina e aldosterone promuovendo la natriuresi;
- la vasopressina, o ormone antidiuretico (ADH), che, in risposta ad aumenti dell'osmolarità plasmatica, determina ritenzione di acqua (Fig. 1).

Questi meccanismi interagiscono tra di loro con il fine ultimo di mantenere costante l'osmolarità plasmatica e i livelli sierici di $\mathrm{Na}^{+}$tra 136-145 mEq/L.

Con il termine ipernatremia si intende una concentrazione di $\mathrm{Na}^{+}$superiore a $145 \mathrm{mEq} / \mathrm{L}$, che si evidenzia clinicamente con manifestazioni a carico del sistema nervoso centrale secondarie alla disidratazione cellulare conseguente all'aumento dell'osmolarità plasmatica. Occorre peraltro sottolineare che il sistema nervoso centrale è in grado di contrastare il raggrinzimento cellulare mediante un meccanismo di "adattamento osmotico", per cui le cellule nervose richiamano dapprima sodio, potassio e aminoacidi e, in seguito, osmoli idiogeniche come inositolo, glutamina e betaina. Ciò fa si che i pazienti con ipernatremia cronica o con ipernatremia a insorgenza molto lenta possano essere relativamente asintomatici.

Obiettivo del presente lavoro non è quello di offrire una completa e dettagliata trattazione della tematica in oggetto, per la quale si rimanda a trattati specialistici, quanto quello di fornire pratiche informazioni sull'approccio diagnosticoterapeutico al paziente con ipernatremia, di utilità nella clinica quotidiana. 
Fig. 1- Meccanismi di autoregolazione dell'osmolarità plasmatica.

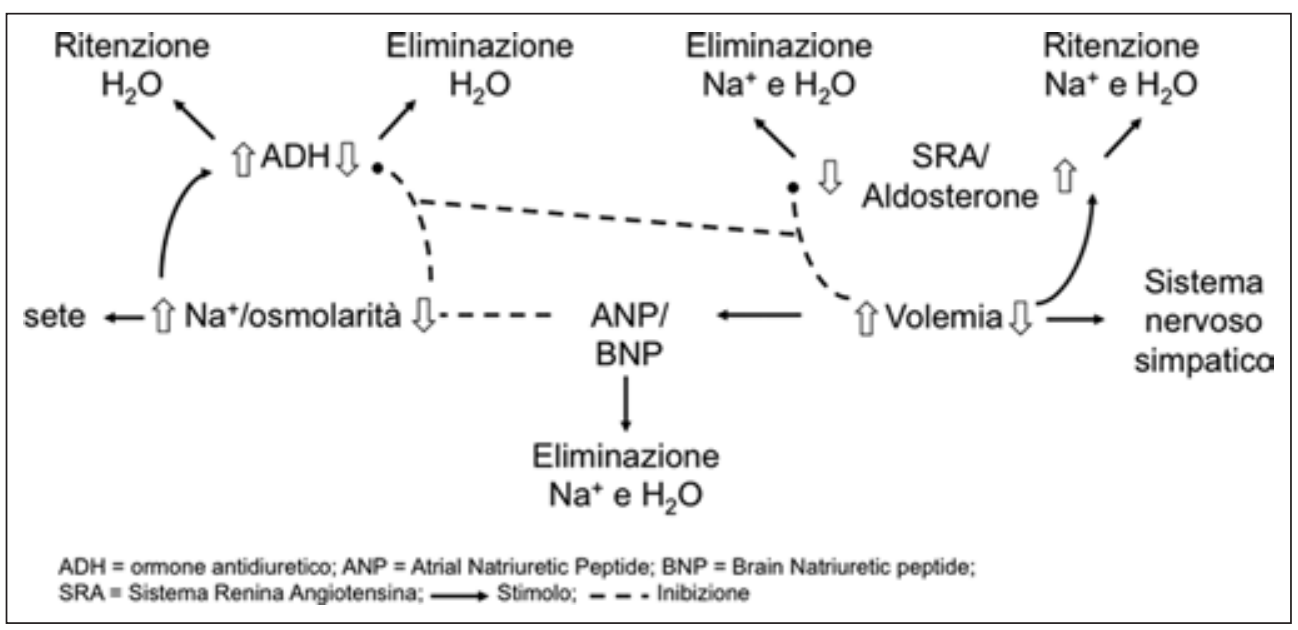

\section{Epidemiologia}

L'ipernatremia rappresenta un disordine elettrolitico che ha un'incidenza di circa il $9 \%$ nei pazienti critici al momento dell'ingresso in ospedale ed è un'alterazione che aumenta significativamente la mortalità intra-ospedaliera di tali pazienti. I fattori di rischio alla base dell'insorgenza di tale disturbo sono l'età avanzata, pregressi danni cerebrali, diabete mellito, interventi chirurgici, terapie diuretiche e alterazioni dello stato mentale. La gran parte dei riscontri di ipernatremia è di natura iatrogena, attribuibile a un'inadeguata terapia idrica in pazienti disidratati e con ridotto stimolo della sete $(1,2)$.

\section{Manifestazioni cliniche}

Le manifestazioni cliniche dell'ipernatremia sono prevalentemente di natura neurologica e sono direttamente correlate alla severità e alla rapidità di insorgenza del disturbo elettrolitico. Sintomi aspecifici come anoressia, irrequietezza, irritabilità, letargia, debolezza muscolare e nausea possono essere presenti negli stadi iniziali; possono manifestarsi confusione e altri segni di alterazione dello stato mentale, aumento dell'eccitabilità neuromuscolare con contrazioni e fascicolazioni, stupore e coma, come conseguenza di un importante e rapido incremento della concentrazione plasmatica di sodio al di sopra di $158-160 \mathrm{mEq} / \mathrm{L}$. L'importanza della sintomatologia dipende dalla gravità dell'iperosmolarità; inoltre, le manifestazioni cliniche delle forme acute risultano essere più marcate di quelle secondarie a un'ipernatremia instauratasi lentamente. L'ipernatremia genera un'intensa sensazione di sete, atta a proteggere le strutture cerebrali dalla disidratazione; questa risposta fisiologica è tuttavia ridotta in pazienti con alterazioni dello stato mentale o con alterazioni ipotalamiche e nei soggetti anziani (3-9).

\section{Eziopatogenesi}

Il quesito da porsi di fronte al riscontro laboratoristico, accompagnato o meno da manifestazioni cliniche, di ipernatremia è valutare se sia realmente aumentato il sodio nel compartimento extracellulare o se sia invece diminuito il solvente, ossia l'acqua. Sulla base di ciò si possono distinguere 3 differenti meccanismi eziopatogenetici le cui cause possono essere riassunte nella Tabella I:

- aumento primitivo del sodio (forma ipervolemica o edematosa);

- mancata introduzione di acqua pura (forma euvolemica);

- perdita di liquidi ipotonici (forma ipovolemica con disidratazione).

\section{Forma clinica ipervolemica o edematosa}

Un introito eccessivo di sodio può essere dovuto a molteplici cause prevalentemente esogene (Tab. I).

In questi casi, l'iperosmolarità che viene indotta determina

\section{TABELLA I - PRINCIPALI CAUSE DI IPERNATREMIA}

Aumento primitivo del sodio (forma ipervolemica)

- infusione di soluzioni ipertoniche di $\mathrm{NaCl}$ e $\mathrm{NaHCO} 3$

- ingestione di acqua marina, sale da cucina

- nutrizione enterale/parenterale

- dialisi ipertonica

- terapia corticosteroidea prolungata

- iperaldosteronismo primitivo

- sindrome di Cushing

Scarso apporto di acqua o perdita di acqua pura (forma euvolemica)

- ipo-adipsia primitiva (malformazioni congenite del SNC, traumi, malattie infettive, malattie cerebrovascolari, neoplasie della neuroipofisi)

- ipo-adipsia secondaria (carenza di acqua, stato mentale alterato, perdita dell'autosufficienza, isolamento)

- diabete insipido centrale/nefrogenico

Perdita di fluidi ipotonici (forma ipovolemica)

- perdite renali (diuretici, diuresi osmotica, diuresi post-ostruttiva, fase poliurica della NTA)

- perdite gastrointestinali (vomito, diarrea osmotica, fistola enterocutanea)

- perdite cutanee (perspiratio insensibilis, ipertermia, sepsi, ustioni, sudorazione profusa)

$\mathrm{SNC}=$ Sistema Nervoso Centrale NTA $=$ Necrosi Tubulare Acuta 


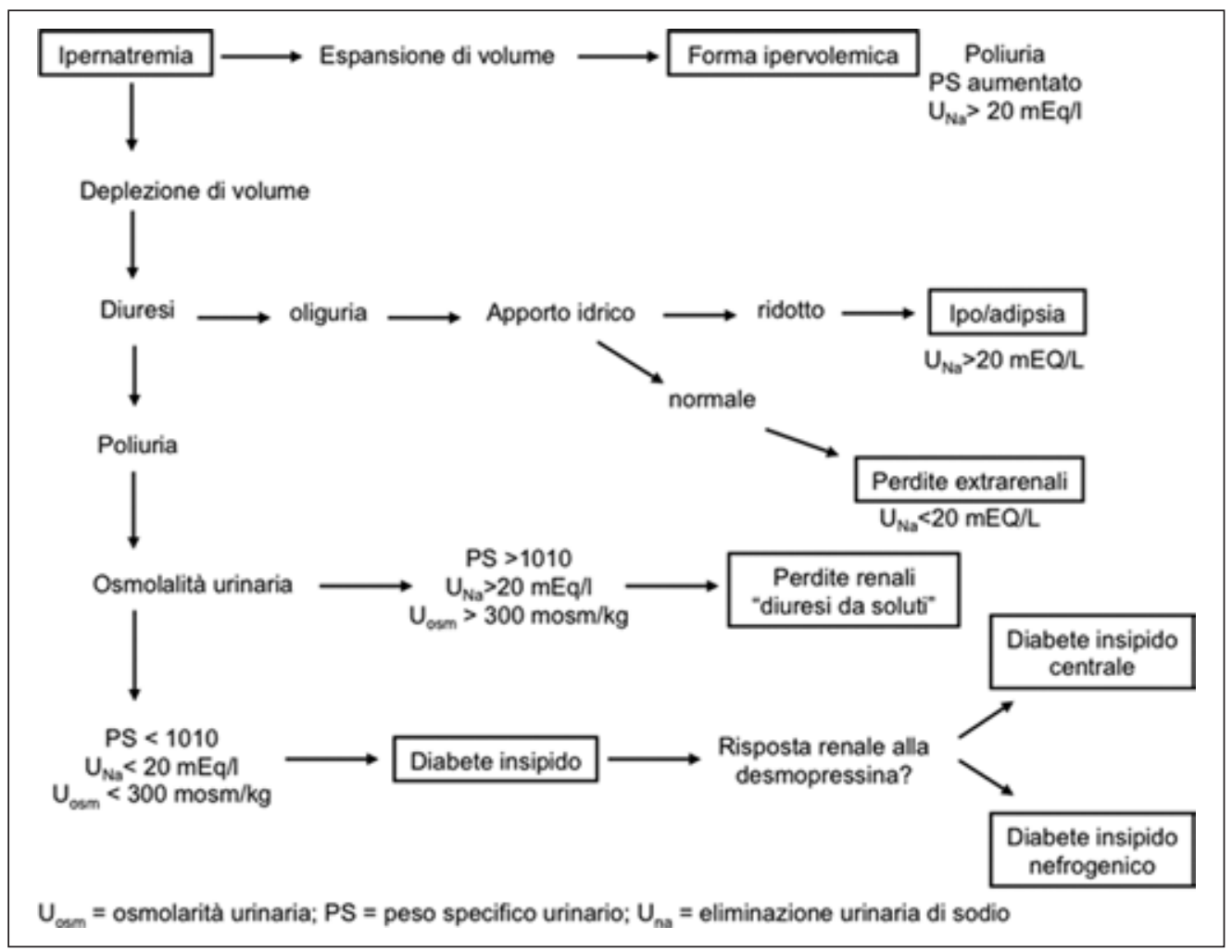

Fig. 2 - Approccio diagnostico all'ipernatremia.

ritenzione di acqua con conseguente formazione di edemi. Laboratoristicamente, oltre a una concentrazione sierica di sodio oltre i limiti di norma, è possibile constatare un'aumentata sodiuria dovuta al sovraccarico plasmatico di sodio, che supera i limiti fisiologici di riassorbimento tubulare. All'incrementata sodiuria concorre anche l'azione dei peptidi natriuretici, in particolare il peptide natriuretico atriale, che, in risposta all'ipervolemia, determina diminuzione del riassorbimento di sodio nel tubulo distale e nel dotto collettore del nefrone, inibizione della secrezione della renina e riduzione della secrezione di aldosterone.

\section{Forme cliniche euvolemiche}

\section{Scarso apporto di acqua}

In questo ambito rientrano tutti i casi di ipo-adipsia, sia primitiva che secondaria (Tab. I). Il quadro clinico è quello classico della disidratazione, con segni di emoconcentrazione, aumento dell'osmolarità plasmatica e urinaria, aumento del peso specifico urinario e aumento dell'ADH e dell'aldosterone. Entrambi questi ormoni, secreti rispettivamente dalla neuroipofisi e dalla corticale surrenale in risposta a condizioni di disidratazione e contrazione del volume ematico, agiscono a livello dei dotti collettori del nefrone, al fine di evitare ulteriori perdite di fluidi e preservare la volemia circolante.

\section{Perdita di acqua pura (diabete insipido)}

Questa forma si caratterizza per la presenza di poliuria a basso peso specifico (diuresi $>3.5$ L con PS $<1010$ ) che può essere tipica del diabete insipido centrale (deficit di secrezione di ADH) e nefrogenico (resistenza all'azione dell'ADH) o della gravidanza (produzione placentare di vasopressinasi).

L'osmolarità urinaria, in questi casi, è inappropriatamente bassa, se paragonata all'aumentata osmolarità plasmatica, con pronto incremento, in caso di diabete insipido centrale, a seguito della somministrazione esogena di ADH.

\section{Forme cliniche ipovolemiche (con disidratazione)}

In questo ambito rientrano le forme cliniche secondarie a perdita di fluidi ipotonici, nelle quali il deficit di acqua è superiore alle perdite elettrolitiche, che possono essere indotte da molteplici fattori eziopatogenetici (Tab. I). Clinicamente, è possibile riscontrare i classici segni e sintomi della disidratazione ipovolemica, come ipotensione marcata, tachicardia con polso piccolo e frequente, cute e mucose secche, papilla da stasi e ipotonia dei bulbi oculari; l'oligo-anuria è un segno clinico caratteristico, eccezion fatta per le forme ipovolemiche secondarie a cause renali. La sodiuria è un parametro che permette di orientare il dubbio diagnostico verso forme gastrointestinali e cutanee (sodiuria $<20 \mathrm{mEq} / \mathrm{L}$ ) o renali (sodiuria $>20 \mathrm{mEq} / \mathrm{L}$ ).

\section{Approccio diagnostico all'ipernatremia (Fig. 2)}

La prima domanda da porsi di fronte a un paziente con ipernatremia è la seguente: è aumentato il sodio totale o è diminuita l'acqua? 
Un corretto approccio diagnostico al reperto laboratoristico di ipernatremia rappresenta un punto chiave nella gestione del paziente e nell'inizio di un trattamento farmacologico adeguato al ripristino di una sodiemia nei limiti della norma.

Innanzitutto, bisogna valutare il volume del compartimento extracellulare andando a ricercare eventuali segni di ipervolemia (edemi periferici) o segni di disidratazione.

- In presenza di edemi periferici si tratterà di un aumento del sodio totale secondario a introito iatrogeno o a malattie surrenaliche (forma clinica ipervolemica).

Se, al contrario, il paziente si presenta disidratato, lo step successivo è la valutazione quantitativa della diuresi:

- In presenza di oliguria (diuresi $<500 \mathrm{cc} /$ die), le urine saranno molto concentrate (PS >1020 e osmolarità >700 mOsm/ $\mathrm{kg})$. A tal riguardo è d'obbligo valutare l'introito idrico:

- se l'oliguria è secondaria a un apporto idrico scarso, la concentrazione urinaria di sodio sarà $>20 \mathrm{mEq} / \mathrm{L}$ e ci troveremo di fronte a un quadro di ipernatremia euvolemica da ipo-adipsia;

- se, invece, l'oliguria si accompagna a un apporto idrico normale, ci sarà una perdita d'acqua prevalentemente per via extrarenale (gastrointestinale, respiratoria, cutanea).

- In presenza di poliuria (diuresi $>2 \mathrm{~L} /$ die), è necessaria un'analisi qualitativa delle urine (PS e osmolarità urinaria):

- se il valore dell'osmolarità urinaria risulta $>300 \mathrm{mOsm} /$ $\mathrm{kg}$ con un PS $>1010$, ci troveremo di fronte a una diuresi da soluti, in quanto il rene non concentra le urine come atteso in una condizione di ipernatremia/iperosmolarità plasmatica (ipernatremia ipovolemica da perdite renali);

- se, al contrario, l'osmolarità urinaria è $<300 \mathrm{mOsm} / \mathrm{kg}$ con un $\mathrm{PS}<1010$ bisognerà indagare su tutte le cause possibili di diabete insipido, differenziando innanzitutto la forma centrale da quella periferica (10). La prima si caratterizza per una mancata produzione di ormone antidiuretico da parte della neuroipofisi ed è secondaria a forme congenite come malformazioni cerebrali o microcefalia o a forme acquisite da distruzione del tessuto neuronale come traumi, infezioni, interventi chirurgici, disordini vascolari, neoplasie e agenti tossici. La seconda si caratterizza, invece, per la mancata risposta delle cellule del tubulo renale alla vasopressina normalmente prodotta e può essere secondaria a forme ereditarie o acquisite come malattie infiammatorie e autoimmuni, patologie vascolari e intossicazione da farmaci. La diagnosi differenziale si basa sul dosaggio plasmatico dell'ormone antidiuretico, indagine peraltro poco eseguita, e sulla risposta urinaria al test di stimolo con desmopressina.

\section{Trattamento}

- Nelle forme di ipernatremia ipervolemica, in presenza di una funzione renale normale, l'eccesso di sodio è generalmente escreto spontaneamente con le urine; la correzione della natremia può essere aumentata dall'infusione di un diuretico dell'ansa, associata a soluzioni isotoniche. In presenza di insufficienza renale acuta o di pre-esistente insufficienza renale cronica (in cui l'eliminazione del sodio è compromessa), deve essere preso in considerazione l'inizio precoce della terapia sostitutiva renale, in particolar modo nei pazienti sintomatici e con valori plasmatici di sodio superiori ai $180 \mathrm{mEq} / \mathrm{L}$ (10). La composizione delle sacche di dialisato comunemente utilizzate nella pratica dialitica presentano una concentrazione di $\mathrm{NaCl}$ pari a $270 \mathrm{~g} / \mathrm{L}$; la diluizione con l'acqua demineralizzata e le impostazioni della conducibilità permettono di ottenere valori di sodiemia nel bagno dialisi in media pari a $140 \mathrm{mEq} / \mathrm{L}$ e ciò permette, attraverso il fenomeno diffusivo, di correggere lo squilibrio elettrolitico del paziente.

- In caso di ipersodiemia da perdita di acqua pura con mantenimento del volume circolante (forma euvolemica), il primo obiettivo è il ripristino della normale tonicità plasmatica con correzione della sodiemia che, peraltro, non deve avvenire in maniera troppo rapida. Il motivo sta nell'adattamento osmotico delle cellule del sistema nervoso centrale, ovvero nel richiamo di osmoli idiogene, che, tuttavia, non abbandonano il citoplasma rapidamente, per cui si potrebbe instaurare un quadro di relativa ipertonicità con conseguente edema cerebrale. Alla luce di questi dati, vanno utilizzate soluzioni relativamente ipotoniche (p. es., soluzione di $\mathrm{NaCl}$ allo $0.45 \%$ ). In letteratura, sono presenti pochi dati in merito alle più sicure modalità di correzione dell'ipernatremia: le attuali raccomandazioni suggeriscono una velocità di correzione dell'ipernatremia non superiore a $1 \mathrm{mEq} / \mathrm{L}$ per ora e di non oltrepassare una diminuzione giornaliera di $10-12 \mathrm{mEq} / \mathrm{L}$. La variazione della sodiemia attesa può essere calcolata secondo la formula:

$$
\begin{aligned}
& \text { variazione della sodiemia = sodio infuso }(\text { con } 1 \mathrm{~L}) \\
& \text { - sodiemia attuale/L di acqua corporea }+1 \mathrm{~L}
\end{aligned}
$$

Per esempio: infondendo $1 \mathrm{~L}$ di soluzione ipotonica allo $0.45 \%$ (77 $\mathrm{mEq})$ in un paziente con sodiemia $163 \mathrm{mEq} / \mathrm{L}$, si ottiene $(77-163) /(42+1)=-2 \mathrm{mEq} / \mathrm{L}$.

In presenza di diabete insipido centrale, la terapia si basa sull'utilizzo di analoghi sintetici della vasopressina (10). Nel diabete insipido nefrogenico è essenziale la cura della patologia renale sottostante o la sospensione degli agenti nefrotossici. Possono essere utilizzati farmaci come diuretici tiazidici o inibitori della sintesi delle prostaglandine (p. es., indometacina), per la capacità di riduzione del volume urinario secondario alla riduzione del flusso ematico renale e della velocità di filtrazione glomerulare.

- In presenza di ipersodiemia ipovolemica, il primo obiettivo è il trattamento dell'emergenza, ovvero il ripristino del volume circolante. A tal riguardo, può essere utilizzata la seguente formula per il calcolo del deficit idrico:

$$
\begin{gathered}
\text { deficit acqua }=(\mathrm{Na} \text { attuale }-\mathrm{Na} \text { desiderato }) \times \text { peso } \\
\text { corporeo } \times 0.6 / \mathrm{Na} \text { desiderato }
\end{gathered}
$$

Per esempio: uomo di $70 \mathrm{Kg}$ con sodiemia $156 \mathrm{mEq} / \mathrm{L}$ :

$$
(156-140) \times 70 \times 0.6 / 140=4.8 \mathrm{~L}
$$

L'infusione terapeutica di fluidi va effettuata inizialmente con soluzioni fisiologiche isotoniche (che, in realtà, sono ipoto- 
niche rispetto al paziente), anche in questo caso a velocità non sostenuta, modulando la concentrazione delle soluzioni alla luce delle determinazioni della sodiemia del paziente (che andrebbe controllata ogni 3 ore) $(11,12)$. Se il paziente è cosciente e con senso della sete non alterato, possono essere somministrati liquidi per via orale, monitorando in maniera costante diuresi e variazioni plasmatiche della natremia.

\section{Conclusioni}

Il reperto laboratoristico di ipernatremia non è esclusivamente sinonimo di aumento della concentrazione plasmatica di sodio, ma è indice di uno squilibrio nel bilancio idrico secondario a perdita o mancata assunzione di acqua, che può avere o meno ripercussioni sul volume circolante. Questo tipo di alterazione elettrolitica, data la scarsità di sintomi specifici, richiede una diagnosi tempestiva e una terapia idonea, in particolar modo nella velocità di somministrazione, per prevenire la morbidità e la mortalità che la caratterizzano.

\section{Riassunto}

I disturbi dell'equilibrio idro-salino risultano estremamente comuni nei pazienti ospedalizzati, in particolar modo in quelli critici. L'ipernatremia, che può essere secondaria a sovraccarico di sodio o a perdita di fluidi ipotonici, può contribuire significativamente alla già importante morbilità e al rischio di morte di questa categoria di pazienti. Questo disturbo richiede pertanto una diagnosi tempestiva e può essere corretto con un intervento appropriato e con il trattamento dei fattori predisponenti che ne sono alla base.

Parole chiave: Ipernatremia, Bilancio idro-salino

Dichiarazione di conflitto di interessi: Gli Autori dichiarano di non avere conflitto di interessi.

Contributi economici agli Autori: Gli Autori dichiarano di non aver ricevuto sponsorizzazioni economiche per la preparazione dell'articolo e per lo svolgimento dello studio.

Indirizzo degli Autori:

Dr. Roberto Di Vito

Istituto di Clinica Nefrologica

Ospedale Clinicizzato

SS. Annunziata

Via dei Vestini

66013 Chieti

divitoroberto@gmail.com

\section{Bibliografia}

1. Bagshaw SM, Townsend DR. Disorder of sodium and water balance in hospitalized patients. J Can Anesth 2009; 56: 151-67.

2. Snyder NA, Feigal DW, Arieff AI. Hypernatremia in elderly patients. A heterogenous, morbid, and iatrogenic entity. Ann Intern Med 1987; 107: 309-19.

3. Palevsky PM, Bhagrath R, Greenberg A. Hypernatremia in hospitalized patients. Ann Intern Med 1996; 124: 197-203.

4. Polderman KH, Schreuder WO, Strack van Schijndel RJ, Thijs LG. Hypernatremia in the intensive care unit: an indicator of quality of care? Crit Care Med 1999; 27: 1105-8.

5. Molaschi M, Ponzetto M, Massaia M, Villa L, Scarafiotti C, Ferrario E. Hypernatremic dehydration in the elderly on admission to hospital. J Nutr Health Aging 1997; 1: 156-60.
6. Hoorn EJ, Betjes MG, Weigel J, Zietse R. Hypernatraemia in critically ill patients: too little water and too much salt. Nephrol Dial Transplant 2008; 23: 1562-8.

7. Lindner G, Funk GC, Schwarz C, et al. Hypernatremia in the critically ill is an independent risk factor for mortality. Am J Kidney Dis 2007; 50: 952-7.

8. Reynolds RM, Padfield PL, Seckl JR. Disorders of sodium balance. BMJ 2006; 332: 702-5.

9. Adrogue HJ, Madias NE. Hypernatremia. N Engl J Med 2000; 342 (20): 1493-9.

10. Sgambato F, Prozzo S, Caporaso C, et al. La Fisiopatologia clinica delle ipersodiemie. Italian Journal of Medicine 2007; 2: 6-18.

11. Offenstadt G, Das V. Hyponatremia, hypernatremia: a physiological approach. Minerva Anestesiol 2006; 72 (6): 353-6.

12. Salvati F. Terapia delle ipersodiemie. Italian Journal of Medicine 2007; 2: 19-23. 